\title{
Quadrupole-free detector optics design for the Compact Linear Collider final focus system at $3 \mathrm{TeV}$
}

\author{
Fabien Plassard, Andrea Latina, Eduardo Marin, and Rogelio Tomás \\ CERN, Geneva, Switzerland \\ Philip Bambade \\ LAL, Orsay, France
}

(Received 29 August 2017; published 17 January 2018)

\begin{abstract}
Aiming to simplify the machine detector interface of the Compact Linear Collider (CLIC), a new detector model has been designed allowing the last quadrupole QD0 of the final focus system (FFS) to be located outside of the experiment with a distance $L^{*}$ from the interaction point of $6 \mathrm{~m}$. In this paper, the beam delivery system (BDS) has been reoptimized, offering a luminosity performance that exceeds the design requirements by $11 \%$ for the total luminosity and by $7 \%$ in the energy peak. A simulation campaign has been carried out and has proved the feasibility of recovering the luminosity under realistic transverse misalignments of the FFS optics, by means of different orbit and aberration correction techniques, making this long $L^{*}$ design a realistic candidate for the future CLIC BDS.
\end{abstract}

DOI: 10.1103/PhysRevAccelBeams.21.011002

\section{INTRODUCTION}

The Compact Linear Collider (CLIC) is an electronpositron linear collider aiming to carry out high-precision lepton physics in the multi-TeV energy range [1]. The nanometer-level beam spot size at the interaction point (IP), required to reach a total luminosity of $5.9 \times 10^{34} \mathrm{~cm}^{-2} \mathrm{~s}^{-1}$, is achieved through the beam delivery system (BDS) [2]. The BDS transports the $e^{+}$and $e^{-}$beams from the exit of the linacs to the IP by performing the critical functions required to meet the CLIC luminosity goal. The beam properties are measured in the diagnostic section at the exit of the linacs and corrected before entering the collimation system. There, the missteered beams coming from the main linac are corrected by the energy collimation system to protect the downstream beam line. The large-amplitude particles, or beam halo, are removed in the transverse collimation section to minimize background in the detector. The beam is then focalized through the final focus system (FFS) while correcting higher-order transport aberrations in order to deliver the design horizontal and vertical beam sizes. The FFS forms an almost parallel beam at the entrance of the two last quadrupoles (QF1 an QD0), referred to as the final doublet (FD), of several hundreds of nanometers which is then demagnified down to a few nanometers at the IP. The beam coming from the main linac is not fully monochromatic, and even a minor energy

Published by the American Physical Society under the terms of the Creative Commons Attribution 4.0 International license. Further distribution of this work must maintain attribution to the author(s) and the published article's title, journal citation, and DOI. spread of a fraction of a percent will cause a large dilution of the beam size at the IP. The chromatic contributions are amplified by the focusing strength of the FD and by the length of the focal distance $L^{*}$ between QD0 and the IP. The chromaticity correction approach chosen for the CLIC FFS is based on the local chromaticity correction scheme [3] which uses interleaved pairs of sextupole magnets in the FD region in order to locally and simultaneously correct horizontal and vertical chromaticity.

The machine detector interface (MDI) design has to satisfy requirements from both the FFS and the detector sides. It must ensure the optimum luminosity for the experiment with minimal backgrounds while meeting constraints from the infrastructure. The FFS baseline for

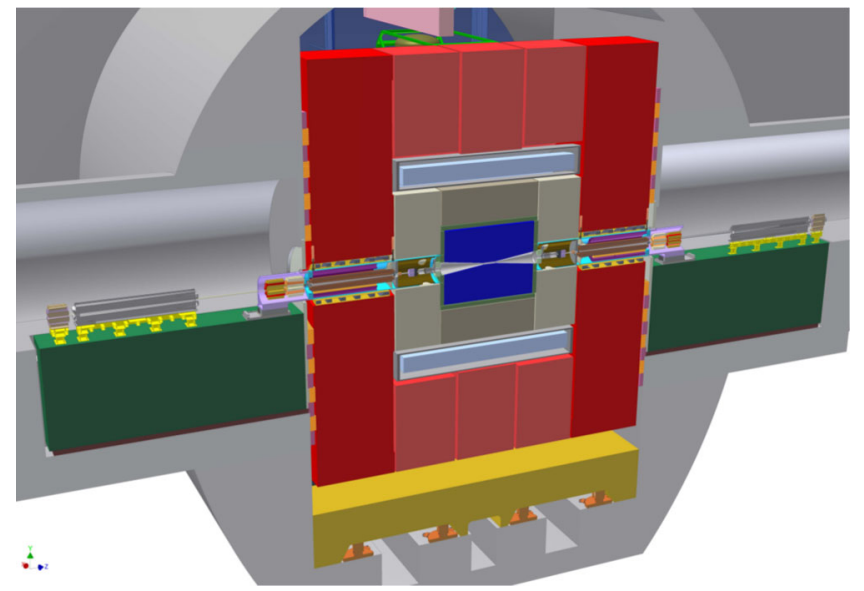

FIG. 1. Vertical cut through the SiD experiment. QD0 is located inside the detector and partially supported by the preinsulator (green block) in the tunnel [4]. 


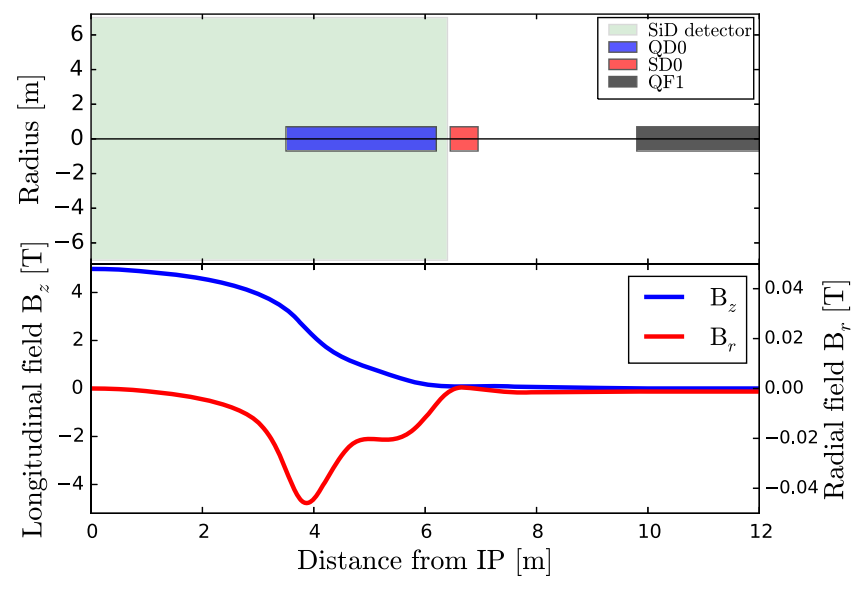

FIG. 2. Schematic overview of the SiD interaction region layout from the last $12 \mathrm{~m}$ of the FFS (upper plot). Simulation of the longitudinal and radial fields (bottom plot). QD0 overlaps with the $\mathrm{SiD}$ solenoid field for $L^{*}=3.5 \mathrm{~m}$.

the conceptual design report (CDR) foresees a short final focal length $L^{*}$ of $3.5 \mathrm{~m}$. This layout places the final quadrupole QD0 inside the experiment as illustrated in Fig. 1 and imposes the integration of a preinsulator system $[4,5]$ and an active insulation to mitigate vibrations of QD0 inside the detector to the $0.1 \mathrm{~nm}$ level. Such a system still needs to be demonstrated in a detectorlike environment. Additionally, due to the presence of a strong magnetic field, higher radiation, and lack of space and access inside the detector, some critical components require longer interventions, leading to a loss of integrated luminosity. The QD0 being installed inside the detector takes away a significant fraction of the acceptance in the forward region. This is partially due to the need of shielding QD0 with an antisolenoid [6,7] in order to reduce the interplay between the detector and the QD0 fields, which would otherwise cause important quadrupole field deterioration and luminosity loss [8]. A schematic overview of the baseline interaction region design together with the simulated solenoid fields expected with the antisolenoid are shown in Fig. 2.

In order to alleviate engineering and stabilization issues of the CDR MDI design, it has been proposed in Refs. $[9,10]$ to move out QD0 from the detector to the tunnel by increasing $L^{*}$. This alternative design features an $L^{*}$ of $8 \mathrm{~m}$, giving $28 \%$ lower luminosity than the nominal design, due to the chromatic distortion which increases with the distance $L^{*}$. Also, the sensitivity to transverse misalignments increased by a factor of 5 [11]. It was then decided to focus the design optimization studies on a FFS with $L^{*}=6 \mathrm{~m}$ which started in Refs. [12,13], delivering a luminosity close to the requirement but with more stringent tolerances against misalignment compared to the nominal design. The CLIC experiments have proposed a new detector model named CLICdet [14], allowing one to move out QD0 from the experiment to the tunnel with a minimum $L^{*}$ of $6 \mathrm{~m}$. The novel interaction region layout is illustrated in Fig. 3. The reduced end cap and barrel yoke give a half

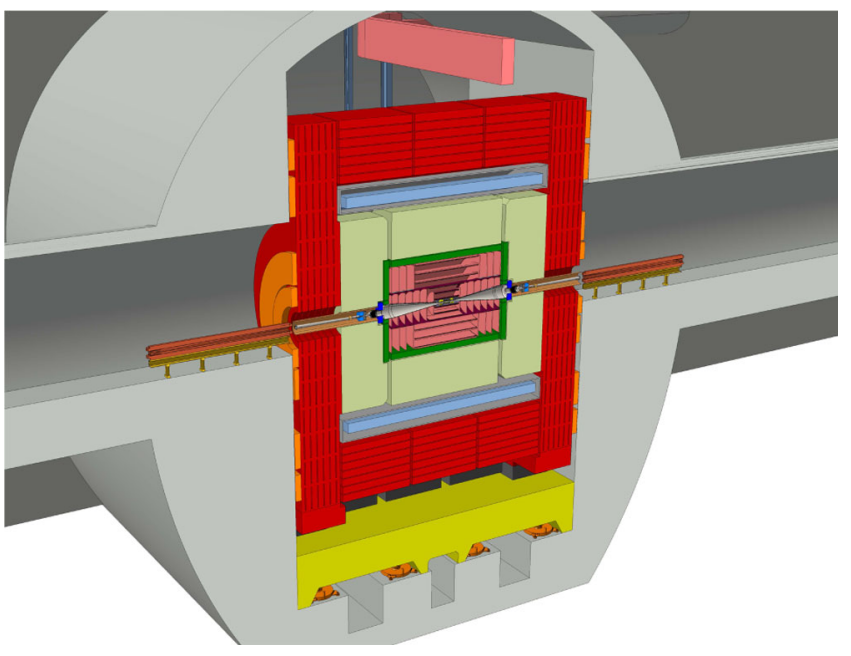

FIG. 3. Vertical cut through the new detector model CLICdet allowing QD0 to be located outside of the experiment. No preinsulator or QD0 shielding are needed as opposed to the short $L^{*}$ design in Fig. 1 [14].

length of the detector of $5918 \mathrm{~mm}$ which includes four ring coils used to remove the solenoid stray fields in the tunnel. The tunnel floor is much more stable than the detector, which will significantly ease the QD0 stabilization $[9,10]$. The preinsulator system is no longer needed, and the access to the detector and QD0 is also simplified. The radial and longitudinal fields of the solenoid of the new detector have been computed along the first $12 \mathrm{~m}$ from the IP and are shown in Fig. 4. The fields are zeroed at the QD0 entrance, and thus no antisolenoid shielding is needed. The IP feedback position and latency are not affected by the change in $L^{*}$ [15]. The new detector and FFS layout should reduce the overall risk, improve the MDI feasibility, and increase the detector acceptance.

In this paper, the impact of the $L^{*}=6 \mathrm{~m}$ FFS option on the luminosity is discussed in Sec. II. Tuning studies have

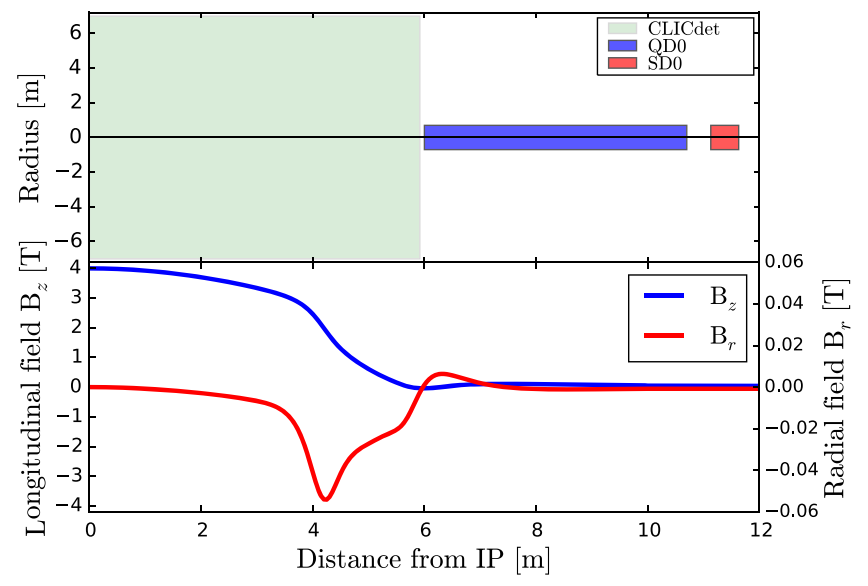

FIG. 4. Schematic overview of the new detector (CLICdet) interaction region layout from the last $12 \mathrm{~m}$ of the FFS (upper plot). Simulation of the longitudinal and radial fields (bottom plot). No overlapping between QD0 and the new detector field with $L^{*}=6 \mathrm{~m}$. 
been carried out to mitigate the effect of static transverse displacements of the quadrupoles, sextupoles, and beam position monitors (BPMs) of the FFS. This procedure utilizes beam-based alignment techniques (BBAs) [16,17], to correct the beam orbit throughout the system, and sextupole tuning knobs, to combat the linear aberrations at the IP. The evaluation of the tuning efficiency is estimated over 100 randomly misaligned machines. Past tuning studies were conducted on the nominal FFS design with $L^{*}=3.5 \mathrm{~m}$ $[2,18]$ and showed that $90 \%$ of the machines should reach $\geq 90 \%$ of the design luminosity in 18000 luminosity measurements. The tuning time needed for the $L^{*}=6 \mathrm{~m}$ FFS to meet the CDR goal has been significantly reduced, and the results are detailed in Sec. III.

\section{NONLINEAR OPTIMIZATION OF THE $L^{*}=6 \mathrm{~m}$ BDS}

The design of the FFS is carried out using the MADX code [19]. The long $L^{*}$ design is based on the FFS scheme called local chromaticity correction [3] starting from the nominal lattice [20] from which the final focal length $L^{*}$ has been increased from 3.5 to $6 \mathrm{~m}$. In order to preserve the chromaticity compensation properties of the beam line, the drifts, dipole, and quadrupole magnets have been scaled in length according to the increase of $L^{*}$. Therefore, the total length of the system has been increased by $71.5 \%$. The quadrupoles have been retuned in order to match the design optics parameters at the IP (see Table I). The optical functions of the $L^{*}=6 \mathrm{~m}$ lattice, compared with the nominal design, are shown in Fig. 5. Longer $L^{*}$ leads to larger $\beta$ functions along the FFS, and the lengthening of the system gives a higher dispersion level.

Six normal sextupoles are used for chromaticity and higher-order aberration corrections. The higher dispersion and $\beta$ functions in the $L^{*}=6 \mathrm{~m}$ design make its sextupoles significantly weaker (see Table II), reducing the contribution from nonlinear aberrations to $\sigma_{x}^{*}$ and $\sigma_{y}^{*}$. However, at a high energy, bending magnets introduce non-negligible growth of the transverse emittance and energy spread due to synchrotron radiation. The angles of the bending magnets

TABLE I. CLIC $3 \mathrm{TeV}$ design parameters.

\begin{tabular}{lcc}
\hline \hline$L^{*}[\mathrm{~m}]$ & 3.5 & 6 \\
\hline FFS length [m] & 450 & 770 \\
Norm. emittance (IP) $\gamma \epsilon_{x} / \gamma \epsilon_{y}[\mathrm{~nm}]$ & $660 / 20$ & $660 / 20$ \\
Beta function (IP) $\beta_{x}^{*} / \beta_{y}^{*}[\mathrm{~mm}]$ & $7 / 0.068$ & $7 / 0.12$ \\
IP beam size $\sigma_{x}^{*} / \sigma_{y}^{*}[\mathrm{~nm}]$ & $40 / 0.7$ & $40 / 0.9$ \\
Bunch length $\sigma_{z}[\mu \mathrm{m}]$ & 44 & 44 \\
rms energy spread $\delta_{p}[\%]$ & 0.3 & 0.3 \\
Bunch population $N_{e}\left[\times 10^{9}\right]$ & 3.72 & 3.72 \\
Number of bunches $n_{b}$ & 312 & 312 \\
Repetition rate $f_{\text {rep }}[\mathrm{Hz}]$ & 50 & 50 \\
Luminosity $\mathcal{L}_{\text {total }}\left[10^{34} \mathrm{~cm}^{-2} \mathrm{~s}^{-1}\right]$ & 5.9 & 5.9 \\
Peak luminosity $\mathcal{L}_{1 \%}\left[10^{34} \mathrm{~cm}^{-2} \mathrm{~s}^{-1}\right]$ & 2 & 2 \\
\hline \hline
\end{tabular}
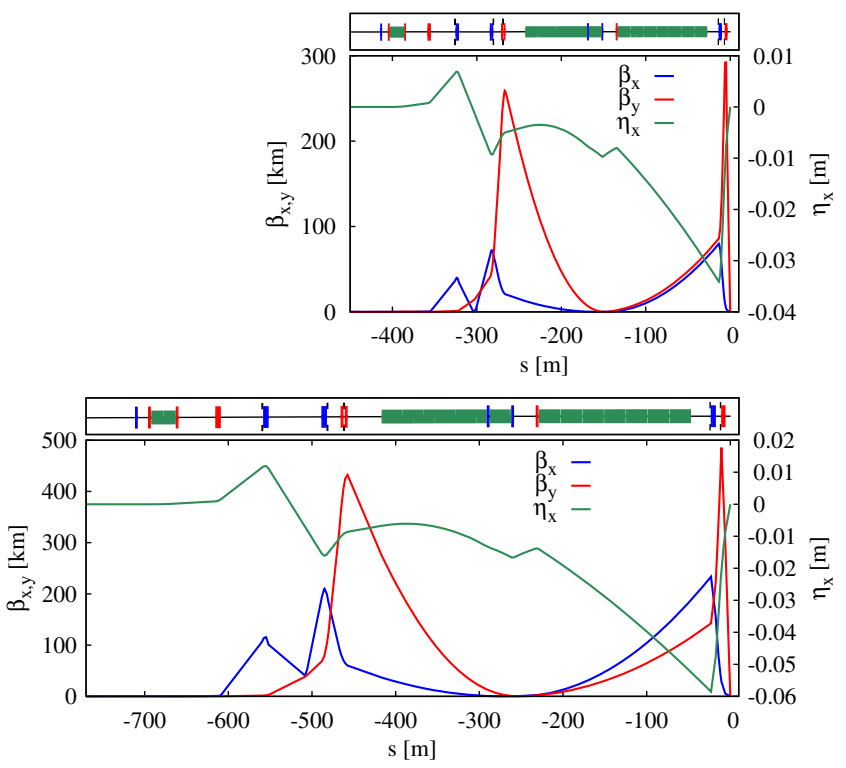

FIG. 5. Optical functions through the local correction scheme of the FFS for $L^{*}=3.5 \mathrm{~m}$ (top plot) and $L^{*}=6 \mathrm{~m}$ (bottom plot), where $\eta_{x}$ is the dispersion function. The lattice for $L^{*}=6 \mathrm{~m}$ has been lengthened with respect to the increase of $L^{*}$ from the nominal design.

have been optimized for the $L^{*}=6 \mathrm{~m}$ FFS in order to balance between these competing effects. The sextupole magnet gradients are tuned to minimize, order by order, the nonlinear contributions to the IP beam size using the MAPCLASS [21-23] and PTC [24] codes.

This nonlinear optimization process has been repeated for several dispersion level options in the FFS. The bending magnet angles have been reduced in order to reduce the average dispersion level at the sextupole locations $\eta_{x}$ up to $40 \%$ with steps of $5 \%$. The beam was tracked through the BDS to the IP using the PLACET code [25], and the total luminosity $\left(\mathcal{L}_{\text {total }}\right)$ and the peak luminosity $\left(\mathcal{L}_{1 \%}\right)$, coming from the collisions with an energy larger than $99 \%$ of the maximum energy, were computed using the GUINEAPIG code [26]. The results are shown in Fig. 6. When lowering the dispersion while taking into account the effects of synchrotron radiation, $\sigma_{y}^{*}$ slowly increases, while $\sigma_{x}^{*}$

TABLE II. Integrated sextupole strengths $k_{2}$, dispersion $\eta_{x}^{s}$, and horizontal $\beta$ function $\beta_{x}^{s}$ at the sextupole locations of the FFS for both optimized $L^{*}$ options.

\begin{tabular}{lccccccr}
\hline \hline & \multicolumn{3}{c}{$L^{*}=3.5 \mathrm{~m}$} & & \multicolumn{3}{c}{$L^{*}=6 \mathrm{~m}$} \\
\cline { 2 - 4 } \cline { 6 - 8 } Magnet & $k_{2}$ & $\eta_{x}^{s}$ & $\beta_{x}^{s}$ & & $k_{2}$ & $\eta_{x}^{s}$ & $\beta_{x}^{s}$ \\
{$[\mathrm{~mm}]$} & {$[\mathrm{km}]$} & & {$\left[\mathrm{m}^{-2}\right]$} & {$[\mathrm{mm}]$} & {$[\mathrm{km}]$} \\
\hline SF6 & 10.5 & 6.5 & 38 & & 3.4 & 12 & 112 \\
SD5 & 19.1 & -4.8 & 20 & & 7.6 & -8 & 55 \\
SF5 & -8.2 & -8.4 & 63 & -2.8 & -15 & 185 \\
SD4 & 16.6 & -5 & 22.3 & & 5.4 & -9 & 66 \\
SF1 & -6.3 & -33 & 79 & & -2.0 & -58.4 & 233 \\
SD0 & 22.5 & -13 & 12 & 7.4 & -22.3 & 34 \\
\hline \hline
\end{tabular}



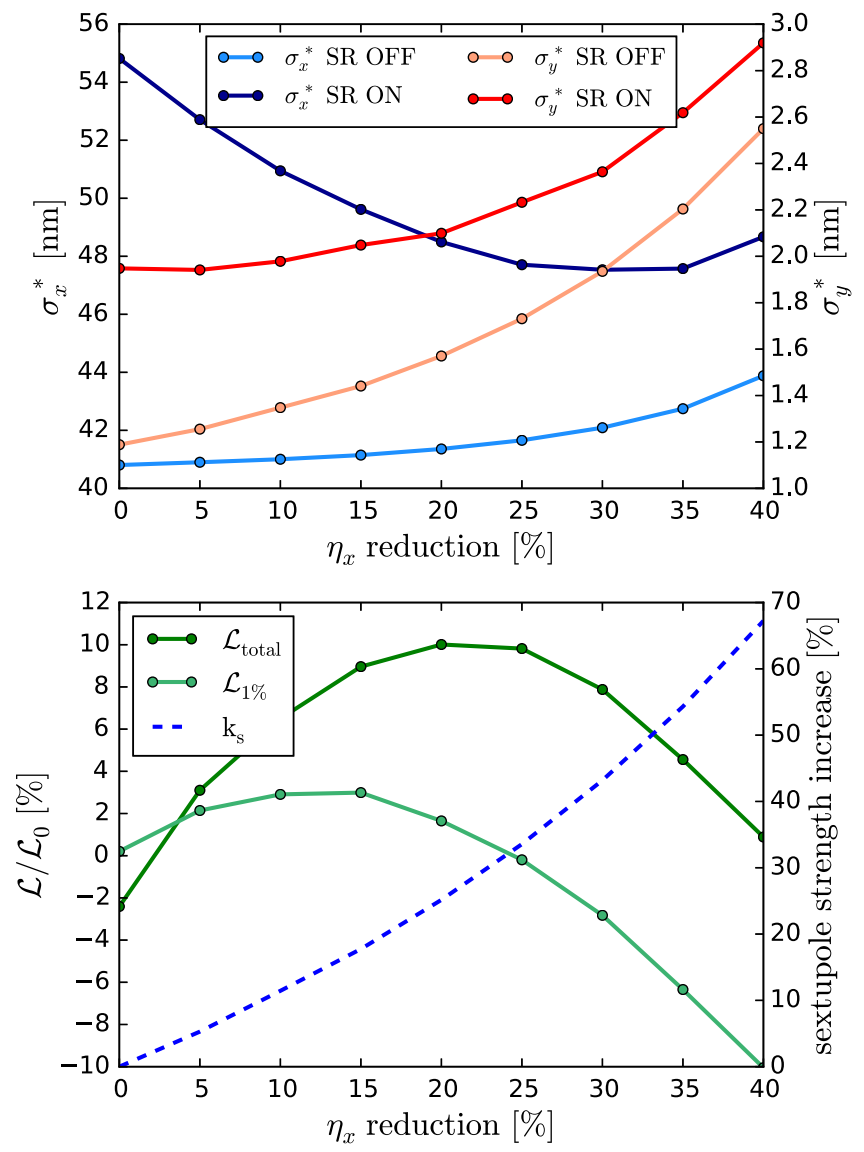

FIG. 6. Impact of the dispersion level in the FFS on the horizontal and vertical beam sizes $\sigma_{x, y}^{*}$ (top plot), luminosity, and sextupole strength $k_{2}$ (bottom plot) when $\eta_{x}$ is reduced up to $40 \%$.

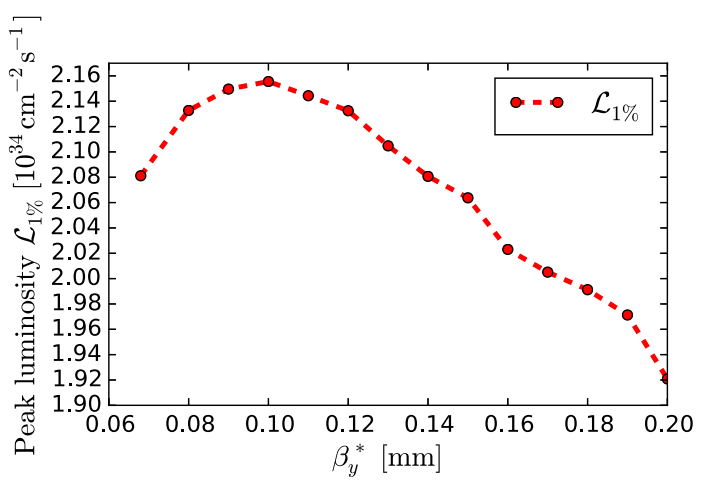

FIG. 7. Peak luminosity maximization versus $\beta_{y}^{*}$ for $L^{*}=6 \mathrm{~m}$. decreases up to a dispersion reduction of $35 \%$. The maximum total luminosity is reached for a lower dispersion than the maximum peak luminosity. This is explained by the increase of beamstrahlung photons emitted for a smaller horizontal beam size that deteriorates the luminosity spectrum in the peak while enhancing the total luminosity [27]. Finally, a dispersion reduction of $15 \%$ has been applied to the FFS, offering an increase of $11 \%$ and $3 \%$ in the total and peak luminosities, respectively. A scan of $\beta_{y}^{*}$ has been performed in order to optimize the luminosity in the peak $\mathcal{L}_{1 \%}$ as shown in Fig. 7. Unlike the nominal $L^{*}$ design [28], $\mathcal{L}_{1 \%}$ reaches a maximum for $\beta_{y}^{*}=0.10 \mathrm{~mm}$. However, $\beta_{y}^{*}=0.12 \mathrm{~mm}$ has been chosen as the design parameter for the tuning efficiency enhancement as described in Sec. III.

The final performance of the long $L^{*}$ is computed for the full BDS, and it is summarized in Table III and compared with the $L^{*}=3.5 \mathrm{~m}$ design. As for the long $L^{*}$ option, the nominal lattice optimized in this study does not include higher-order multipoles, such as octupoles or decapoles, and differs therefore slightly from the performances exposed in Ref. [20]. The vertical chromaticity generated through the entire FFS, given in Table III, was computed using the definition of the chromaticity derived from the transfer map between two locations in the beam line:

$$
\vec{x}_{f}=\sum_{j k l m n} \vec{X}_{j k l m n} x_{0}^{j} p_{x 0}^{k} y_{0}^{l} p_{y 0}^{m} \delta_{0}^{n}
$$

and given by [13]

$$
\xi_{y}^{* 2}=\left(X_{00101}^{2} \frac{\beta_{y 0}}{\beta_{y}^{*}}+X_{00011}^{2} \frac{1}{\beta_{y 0} \beta_{y}^{*}}\right),
$$

where $\vec{X}_{j k l m n}$ is the map coefficient between the initial and the final coordinates represented by $\vec{x}_{f}=\left(x_{f}, p_{x f}, y_{f}\right.$, $\left.p_{y f}, \delta_{f}\right)$ and $\beta_{y 0}$ is the initial vertical $\beta$ function.

The total and peak luminosities of the optimized $L^{*}=$ $6 \mathrm{~m}$ design are reduced by $7.7 \%$ and $7 \%$, respectively, compared to the nominal $L^{*}$ design, without higher-order multipoles included in the beam line. However, when octupoles and decapoles are optimized in the $L^{*}=$ $3.5 \mathrm{~m}$ beam line [20], the difference in $\mathcal{L}_{\text {total }}$ and $\mathcal{L}_{1 \%}$ between both $L^{*}$ options is $11.5 \%$ and $10.7 \%$, respectively. The luminosity loss due to the possible energy mismatch

TABLE III. Simulated performance of both $L^{*}$ options when the beam is tracked through the entire BDS (for $L^{*}=6 \mathrm{~m}, \beta_{y}^{*}=0.12 \mathrm{~mm}$ ). Comparison of the vertical chromaticity, total and peak luminosities, and impact of synchrotron radiation at the IP.

\begin{tabular}{lcccccc}
\hline \hline Design & $\xi_{y}^{*}$ & $\frac{\sigma_{x}^{*}}{\sigma_{x}^{\text {*noSR }}}$ & $\frac{\sigma_{y}^{*}}{\sigma_{y}^{* \text { noSR }}}$ & $\mathcal{L}_{\text {total }}\left[10^{34} \mathrm{~cm}^{-2} \mathrm{~s}^{-1}\right]$ & $\mathcal{L}_{1 \%}\left[10^{34} \mathrm{~cm}^{-2} \mathrm{~s}^{-1}\right]$ & $\frac{\mathcal{L}_{1 \%}}{\mathcal{L}_{1 \%}^{\text {noSR }}}$ \\
\hline$L^{*}=3.5 \mathrm{~m}$ & 82027 & 1.18 & 1.86 & 7.04 & 2.3 & 0.81 \\
$L^{*}=6 \mathrm{~m}$ & 79913 & 1.21 & 1.35 & 6.5 & 2.14 & 0.88 \\
\hline \hline
\end{tabular}




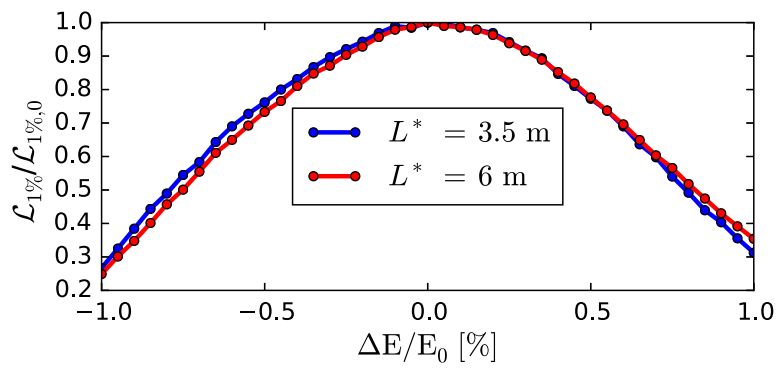

FIG. 8. Energy bandwidth comparison between $L^{*}=3.5 \mathrm{~m}$ and $L^{*}=6 \mathrm{~m}$. $\mathcal{L}_{1 \%}$ is normalized to their respective maximum peak luminosity $\mathcal{L}_{1 \%, 0}$.

coming from the linac is shown in Fig. 8. The energy bandwidth is similar for both optimized $L^{*}$ options. The FD parameter comparisons between both $L^{*}$ options are summarized in Table IV.

\section{FFS TUNING}

The FFS quadrupoles, sextupoles, and BPMs are randomly misaligned by $\sigma_{\mathrm{rms}}=10 \mu \mathrm{m}$, according to the prealignment specification [2,29] for the CLIC beam delivery system. A resolution of $10 \mathrm{~nm}$ is applied to the BPMs used for orbit correction. The assumed imperfections for the tuning simulations are summarized in Table V. The BBA starts with a one-to-one correction technique [30] that aims to flatten the orbit by steering the beam through the center of each BPM using transverse kickers. The effectiveness of the orbit correction is compromised by the misaligned beam position monitors, leading to a dispersive orbit. A technique called dispersion-free steering [31] removes the remaining dispersion deviations from the nominal dispersion profile. Linear aberrations at the IP created by the misaligned optics are corrected using precomputed combinations of sextupole displacements in the transverse plane $[32,33]$. Each set of sextupole knobs is

TABLE IV. Final doublet gradient and aperture radius comparison.

\begin{tabular}{lcc}
\hline \hline FD parameters & $L^{*}=3.5 \mathrm{~m}$ & $L^{*}=6 \mathrm{~m}$ \\
\hline$G_{\mathrm{QF} 1}[\mathrm{~T} / \mathrm{m}]$ & 202.4 & 68.6 \\
$G_{\mathrm{QD} 0}[\mathrm{~T} / \mathrm{m}]$ & -581.5 & -197 \\
$\mathrm{Ap}_{\mathrm{QF} 1}[\mathrm{~mm}]$ & 7.4 & 22 \\
$\mathrm{Ap}_{\mathrm{QD} 0}[\mathrm{~mm}]$ & 3.8 & 8 \\
$l_{\mathrm{QF1} 1}[\mathrm{~m}]$ & 3.26 & 5.6 \\
$l_{\mathrm{QD0}}[\mathrm{m}]$ & 2.73 & 4.7 \\
\hline \hline
\end{tabular}

TABLE V. rms errors applied to the FFS lattice.

$\sigma_{\text {offset }}$ (quadrupole, sextupole, and BPMs)

$10 \mu \mathrm{m}$

$\mathrm{BPM}$ resolution

$10 \mathrm{~nm}$

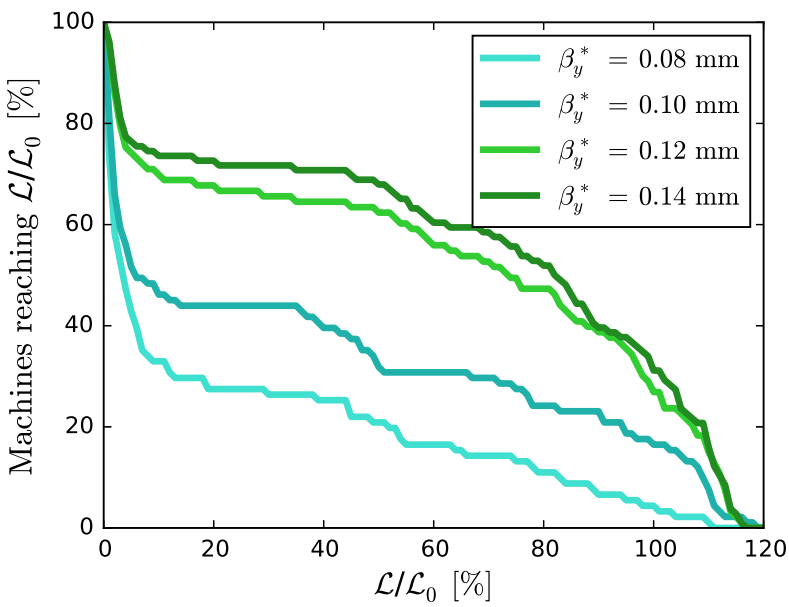

FIG. 9. Luminosity distribution after BBA and one scan of the linear knobs for different values of $\beta_{y}^{*} .100$ randomly misaligned machines are simulated, and the luminosity is normalized to the design luminosity $\mathcal{L}_{0}=5.9 \times 10^{34} \mathrm{~cm}^{-2} \mathrm{~s}^{-1}$.

constructed to be orthogonal, so that the chosen aberrations are corrected independently.

The BBA and one iteration of linear knobs have been applied to the $L^{*}=6 \mathrm{~m}$ FFS for different $\beta_{y}^{*}$ options. Here one iteration of the linear knobs includes two optimizations of each knob. Larger $\beta_{y}^{*}$ allows lower $\beta_{y}$ along the FFS, making the system more robust to transverse errors. Figure 9 shows the luminosity distribution of the 100 machines tuned for different $\beta_{y}^{*}$ options. The luminosity is

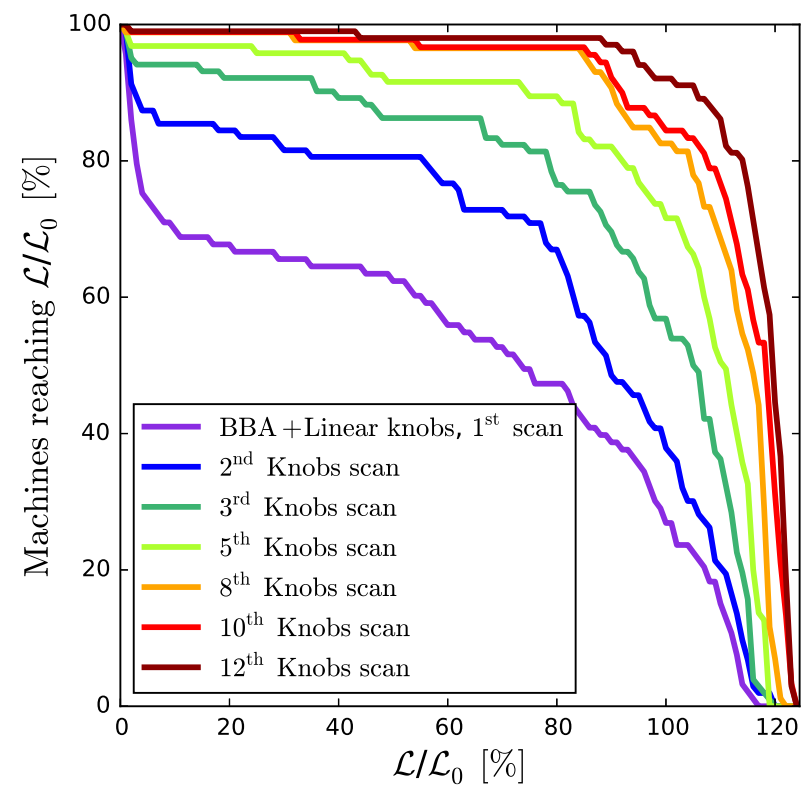

FIG. 10. Tuning performance results for the optimized $L^{*}=6 \mathrm{~m}$ design with $\beta_{y}^{*}=0.12 \mathrm{~mm}$. $87 \%$ of the machines achieve at least $110 \%$ of the design luminosity after 12 iterations of linear knobs, corresponding to approximately 5000 luminosity measurements. 
TABLE VI. Vertical offset tolerances (in nanometers) for the last quadrupole magnets in the CLIC FFS for a relative peak luminosity loss of $2 \%$.

\begin{tabular}{lcc}
\hline \hline Magnet & $L^{*}=3.5 \mathrm{~m}$ & $L^{*}=6 \mathrm{~m}$ \\
\hline QD0 & 0.2 & 0.25 \\
QF1 & 0.8 & 1 \\
QD2 & 8 & 9 \\
QF3 & 16 & 19 \\
\hline \hline
\end{tabular}

normalized to the design luminosity $\mathcal{L}_{0}$. Large $\beta_{y}^{*}$ have a positive impact on the tuning performance. The number of machines that reach $90 \%$ of $\mathcal{L}_{0}$ has been increased from $22 \%$ to $39 \%$ when $\beta_{y}^{*}$ is increased from 0.1 to $0.12 \mathrm{~mm}$, while the luminosity loss is very small compared to the gain in tuning efficiency. This justifies the design $\beta_{y}^{*}$ in Table I for $L^{*}=6 \mathrm{~m}$.

Only linear knobs are then applied iteratively in order to maximize the luminosity of each machine. Figure 10 shows the evolution of the luminosity distribution after various iterations of knob scan. The increase of the average luminosity recovered is fast for the first iterations and starts to slowly converge for a higher number of knob scans. After the 12th iteration, which corresponds to $\approx 5000$ luminosity measurements, $97 \%, 92 \%$, and $87 \%$ of the machines reach $\geq 90 \%, 100 \%$, and $110 \%$ of $\mathcal{L}_{0}$, respectively. These results demonstrate the tuning feasibility of the $L^{*}=6 \mathrm{~m}$ FFS design to be at the same level or better than the one of the $L^{*}=3.5 \mathrm{~m}$ in the CDR [2].

\section{QUADRUPOLE STABILIZATION TOLERANCES}

An important source of luminosity loss for CLIC is related to the change of the $e^{+}$and $e^{-}$beam positions at the IP due to quadrupole position jitter. The vibration error tolerances are tighter in the vertical plane due to the smaller beam size. The tolerances in the FD are expected to be the tightest, as a vertical displacement of the FD causes a displacement of the beam at the IP of the same magnitude. The vertical vibration tolerances have been quantified for the last four FFS quadrupoles QF3, QD2, QF1, and QD0 of the $L^{*}=6 \mathrm{~m}$ lattice. The results are compared, in Table VI, with the nominal FFS design where the tolerances have been studied in Ref. [34]. The tolerances are defined as the offset required to induce a $2 \%$ peak luminosity loss and do not take into account the correction of the beam offset at the IP by the IP position feedback. A comparison of the vertical offset scan performed on QD0 for $L^{*}=6 \mathrm{~m}$ and $L^{*}=3.5 \mathrm{~m}$ is shown in Fig. 11. As the vertical beam size $\sigma_{y}^{*}$ is larger for the $L^{*}=6 \mathrm{~m}$ option, its tolerances to vibration errors in its final quadrupole magnets are therefore slightly larger.

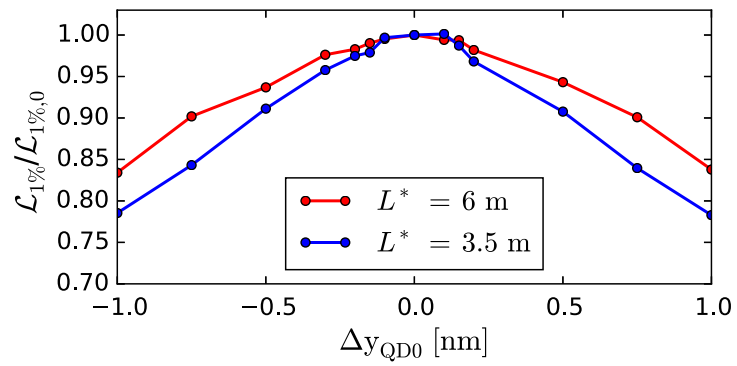

FIG. 11. QD0 vertical offset scan as a function of the relative peak luminosity loss for $L^{*}=6 \mathrm{~m}$ and $L^{*}=3.5 \mathrm{~m}$ designs.

\section{FD MULTIPOLE FIELD TOLERANCES}

The multipolar tolerances are defined as the error required to induce a $2 \%$ peak luminosity loss. The tolerances are evaluated for the FD, where they are the tightest, and compared between the nominal and the long $L^{*}$ designs. The tolerances to normal and skew sextupole, octupole, and decapole field errors, denoted $B_{3 \mathrm{~N}}, B_{4 \mathrm{~N}}, B_{5 \mathrm{~N}}$, $B_{3 \mathrm{~S}}, B_{4 \mathrm{~S}}$, and $B_{5 \mathrm{~S}}$, are calculated individually for $\mathrm{QF} 1$ and QD0 as shown in Figs. 12 and 13. The multipolar magnetic field errors are applied at a radius $R=1 \mathrm{~mm}$. The larger $\beta_{x, y}$ at the FD location for the $L^{*}=6 \mathrm{~m}$ design makes the
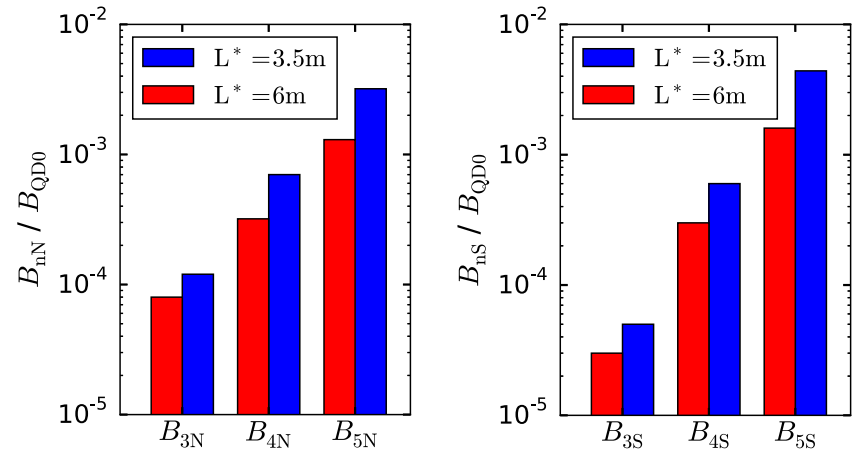

FIG. 12. Normal (left plot) and skew (right plot) sextupole, octupole, and decapole field error tolerances for QD0.
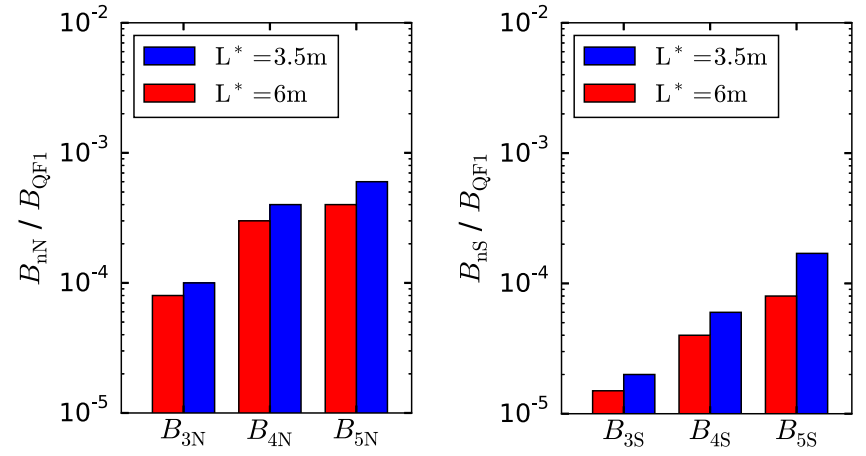

FIG. 13. Normal (left plot) and skew (right plot) sextupole, octupole, and decapole field error tolerances for QF1. 
lattice more sensitive to higher multipole field errors compared to the nominal design.

\section{COLLIMATION DEPTH}

The BDS collimator apertures were optimized for the CLIC FFS at $3 \mathrm{TeV}$ with $L^{*}=3.5 \mathrm{~m}$ with the aim of mitigating the wakefield effects on the luminosity performance while keeping a good efficiency in cleaning the undesired beam halo. The betatron collimation depths have to satisfy the condition that no beam particles or synchrotron radiation photons should hit either QF1, QD0, the vertex detector, or the extraction quadrupoles. For CLIC, the tight bore aperture of the final quadrupole QD0 determines the actual collimation depth. For the $L^{*}=$ $3.5 \mathrm{~m}$ FFS design, QD0 imposes an aperture of $3.8 \mathrm{~mm}$. The optimum transverse collimation depths were found for $15 \sigma_{x}$ and $55 \sigma_{y}$ [2]. The beam size at the entrance of the FD for $L^{*}=6 \mathrm{~m}$ is approximately $30 \%$ larger than the nominal design due to the increase of $L^{*}$. The lower field gradient of QD0, imposed by the longer focal length $L^{*}$ and the longer magnet length $l_{\mathrm{QD} 0}$ after the scaling of the FFS length with respect to the increase of $L^{*}$, allows an aperture for QD0 of $8 \mathrm{~mm}$ (see Table IV). In this study, the impact of the new $L^{*}=6 \mathrm{~m}$ design on the necessary collimator apertures is compared to the nominal settings. The beam is assumed to be perfectly collimated at the entrance of the FFS with a phase space ellipse thickness of $15 \sigma_{x}, 15 \sigma_{x^{\prime}}, 55 \sigma_{y}$, and $55 \sigma_{y^{\prime}}$. The beam is then tracked through the FFS with a flat energy spread distribution of width $1.4 \%$ and by taking into account synchrotron radiation along the beam line. The same simulation has been reproduced for the nominal design for comparison. The collimated beam distribution at the entrance of the FFS is shown in Fig. 14. The transverse beam distributions were simulated at different locations through QF1 and QD0, and the photons emitted were tracked from the entrance of the FD to the exit of the

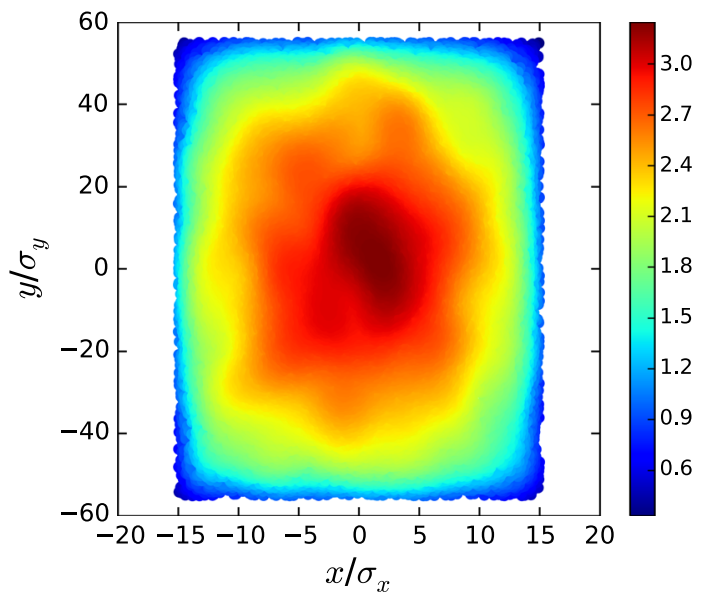

FIG. 14. Halo distribution after collimation to $15 \sigma_{x}$ and $55 \sigma_{y}$ at the entrance of the FFS.
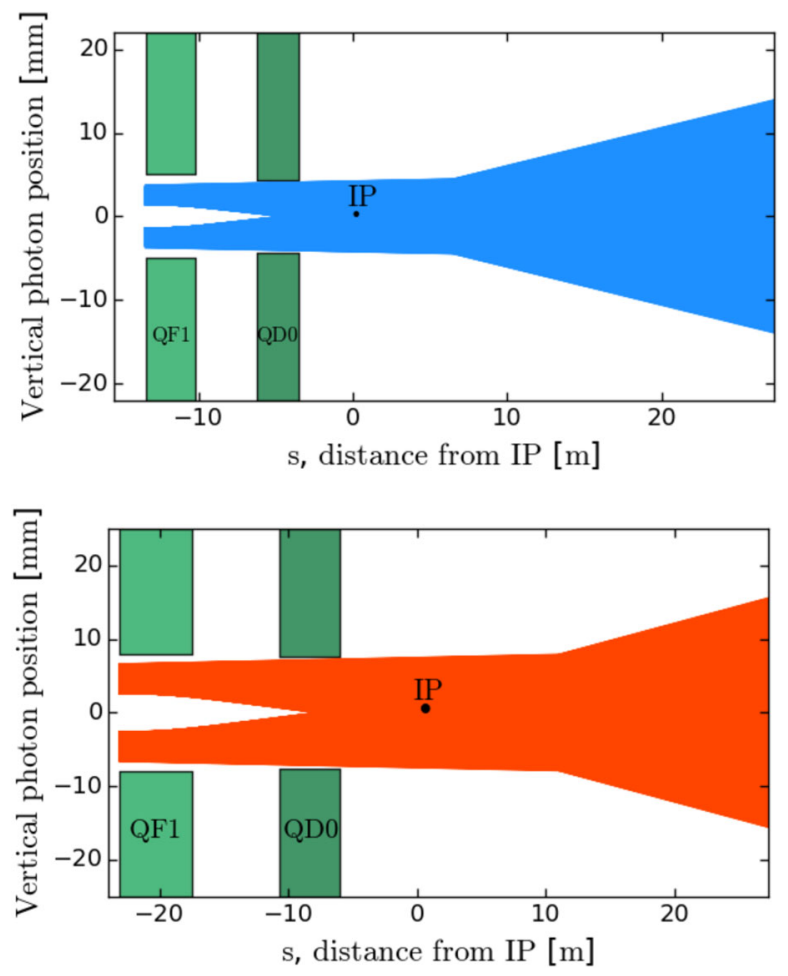

FIG. 15. Horizontal position of the emitted photons through the FD and CLIC detector, up to the entrance of the first postcollision magnet, for $L^{*}=3.5 \mathrm{~m}$ (top plot) and $L^{*}=6 \mathrm{~m}$ (bottom plot).
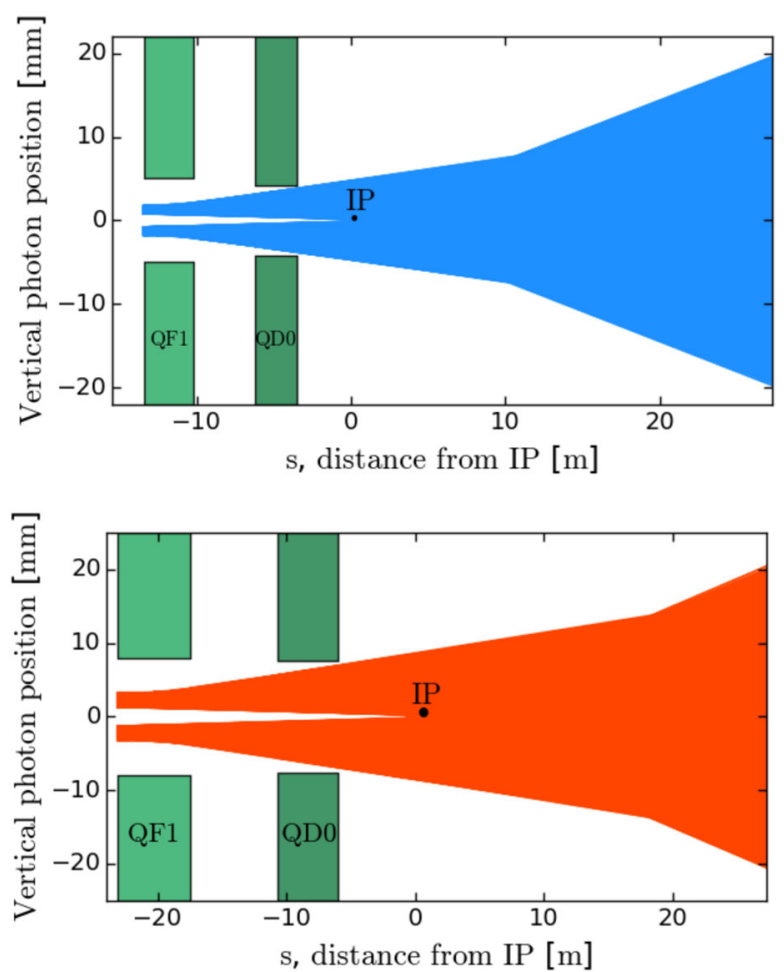

FIG. 16. Vertical position of the emitted photons through the FD and CLIC detector, up to the entrance of the first postcollision magnet, for $L^{*}=3.5 \mathrm{~m}$ (top plot) and $L^{*}=6 \mathrm{~m}$ (bottom plot). 
postcollision magnets. Starting at the IP, the spent beam leaves the detector region through $27.5 \mathrm{~m}$ of drift space [2] before traversing five vertically bending magnets. The results are shown in Figs. 15 and 16 and show that the beam particles and emitted photons pass safely through the FD. The inner radius of the IP beam pipe is $29.4 \mathrm{~mm}$ [4], and the simulations indicate that the radiation envelopes stay within $\pm 5 \mathrm{~mm}$ for $L^{*}=3.5 \mathrm{~m}$ and $\pm 10 \mathrm{~mm}$ for $L^{*}=6 \mathrm{~m}$. Also, the apertures of the postcollision magnets, reported in the CDR [2], are large enough to allow the passage of the simulated synchrotron radiation fan. The results suggest that no tightening of the collimation depth is required for the scaled in length $L^{*}=6 \mathrm{~m}$ FFS design.

\section{FFS LENGTH OPTIMIZATION}

The long $L^{*}$ design presented in Sec. II is $320 \mathrm{~m}$ longer than the nominal FFS lattice with $L^{*}=3.5 \mathrm{~m}$, requiring an extension of the CLIC tunnel. In the interest of cost reduction, shorter FFS lattices have been optimized. The design optimization procedure applied to these lattices, their performances, and the impact on tuning are discussed in this section.

\section{A. Shorter FFS with $L^{*}=6 \mathrm{~m}$}

A shorter FFS has been designed by shortening drifts, bending magnets, and quadrupole magnets proportionally while keeping the last drift $L^{*}$ at a constant length. Therefore, the chromatic correction conditions through the FFS to the IP are no longer satisfied, leading to additional uncorrected second-order horizontal chromatic aberrations. After reducing the length of the FFS $\left(L_{\mathrm{FFS}}\right)$ with $L^{*}=6 \mathrm{~m}$, the linear optics are rematched and the sextupoles optimized order by order using the MAPCLASS code. The second-order horizontal beam size growth due to the change in the lattice length is shown in Fig. 17. The tracking of a monochromatic beam, $\delta_{p}=0$, shows that the

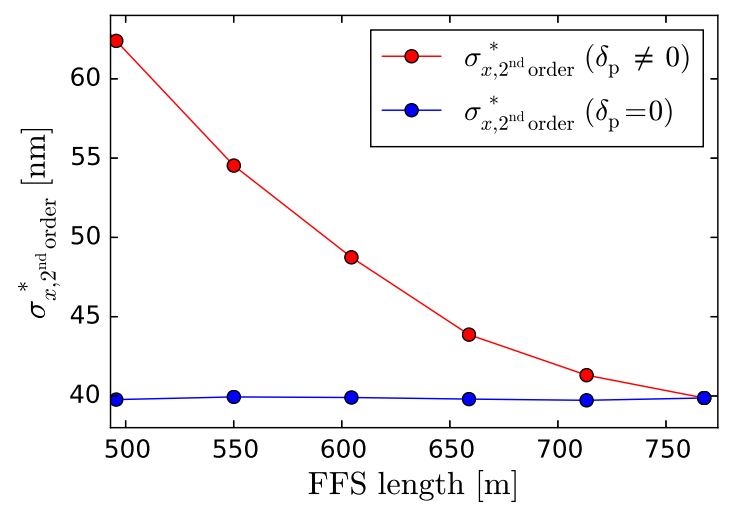

FIG. 17. Horizontal beam size $\sigma_{x}^{*}$ including only second-order aberrations, simulated before $L_{\mathrm{QF} 1-\mathrm{QD} 0}$ optimization, as a function of the FFS length.

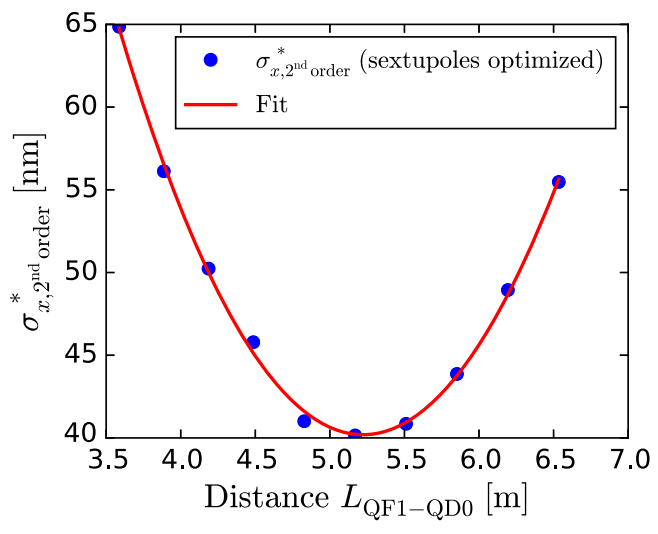

FIG. 18. Example of $L_{\mathrm{QF1}-\mathrm{QD} 0}$ scan for $\sigma_{x, 2 \text { nd order }}^{*}$ minimization applied on the $L_{\mathrm{FFS}}=658.9 \mathrm{~m}$ lattice. The original distance $L_{\mathrm{QF1} 1-\mathrm{QD} 0}$ after FFS length reduction was $5.8 \mathrm{~m}$.

impact of $L_{\mathrm{FFS}}$ reduction on the second-order horizontal beam size $\sigma_{x, 2 \text { nd order }}^{*}$ is fully chromatic.

In order to rebalance the chromatic compensation and therefore minimize the horizontal second-order chromatic aberrations, the chromaticity generated by the FD has been optimized by scanning the distance $L_{\mathrm{QF1} 1 \mathrm{QD} 0}$ between QF1 and QD0 [35]. When the FFS is shortened with constant $L^{*}$, the chromaticity is overcompensated downstream of the bends. The $L_{\mathrm{QF1} \text {-QD0 }}$ has thus to be shortened in order to reduce the chromaticity generated by the FD. Figure 18 shows an example of $L_{\mathrm{QF1} \text {-QD0 }}$ scan for $\sigma_{x, 2 \text { nd order }}^{*}$ minimization applied to a lattice length reduced from 770 to $658.9 \mathrm{~m}$. The scan is performed by rematching the linear optics and optimizing the sextupoles for every change in $L_{\mathrm{QF1}-\mathrm{QD} 0}$. The optimal distance $L_{\mathrm{QF1} 1-\mathrm{QD} 0}$ is found by fitting a parabola to the obtained $\sigma_{x, 2 \text { nd order }}^{*}$ The scan has been repeated on five different lattices with $L_{\mathrm{FFS}}$ reduced down to $496.7 \mathrm{~m}$. The fits of the scans for all lattices are shown in Fig. 19. The horizontal second-order chromatic aberrations are fully corrected after $L_{\mathrm{QF1}-\mathrm{QD} 0}$ optimization, and $\sigma_{x, 2 \mathrm{nd} \text { order }}^{*}$ is approximately $40 \mathrm{~nm}$ for all designs. The

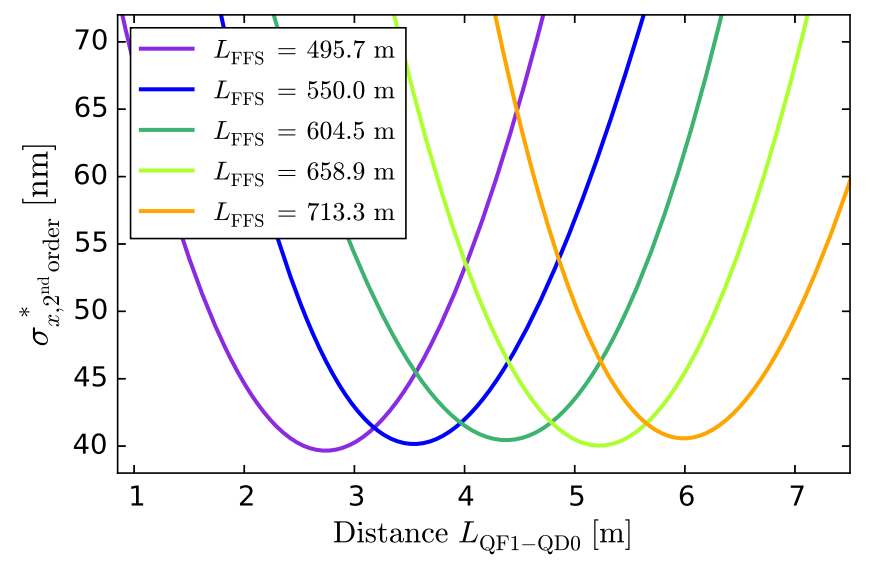

FIG. 19. Parabolic fits of the $L_{\mathrm{QF} 1-\mathrm{QD} 0}$ scan for different FFS lengths. 


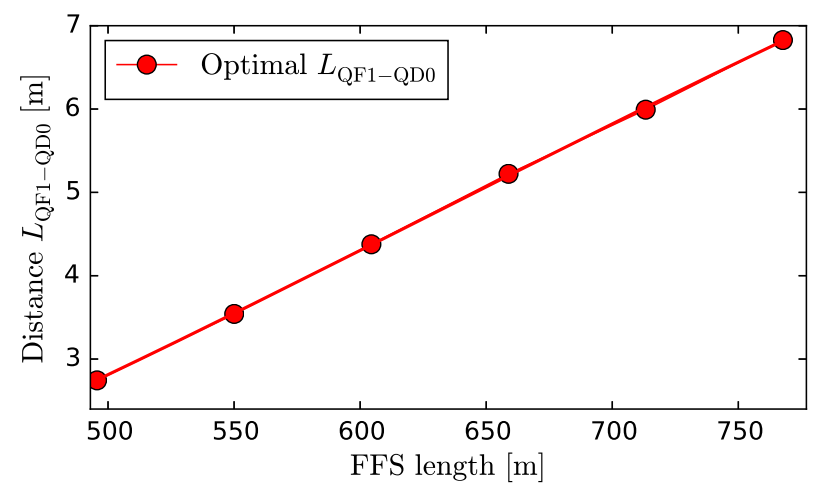

FIG. 20. Optimal distance $L_{\mathrm{QF} 1-\mathrm{QD} 0}$ for $\sigma_{x, 2 \text { nd order }}^{*}$ minimization as a function of the FFS length.

optimal distances $L_{\mathrm{QF1} \text {-QD0 }}$ decreases linearly with $L_{\mathrm{FFS}}$ reduction as shown in Fig. 20.

The sextupoles of the shorter lattices have been retuned to cancel higher-order aberrations. The dispersion has been optimized by scanning the bend angles in the FFS in order to maximize $\mathcal{L}_{\text {total }}$ and $\mathcal{L}_{1 \%}$. The final performance of each lattice is presented in Table VII. Except for the shortest design simulated with $L_{\mathrm{FFS}}=496.7 \mathrm{~m}$, for which the peak luminosity is $1.5 \%$ below the design, all lattices reach or exceed the required luminosity (see Table I). By shortening the bending magnets of the FFS, the observed impact of synchrotron radiation on the horizontal beam size $\sigma_{x}^{*}$ is slightly reduced. However, as the quadrupole lengths are scaled, the synchrotron radiation inside the FD becomes important for shorter lattices. The shorter and stronger QD0 for shorter designs increases the vertical beam size contribution from the Oide effect $[36,37]$. The vertical beam size contribution from the Oide effect $\sigma_{y \text {,Oide }}$ has been calculated using the MAPCLASS code and is shown in Table VII. Finally, the impact of synchrotron radiation on the peak luminosity increases when the length of the system is reduced. As QF1 and QD0 are stronger for shorter designs with constant $L^{*}$, their maximum apertures are smaller, and therefore collimation depth may be a concern.

\section{B. Shorter FFS impact on tuning}

The tuning algorithm described in Sec. III has been applied on different FFS length designs with $L^{*}=6 \mathrm{~m}$.
The tuning efficiency is expected to be reduced for shorter lattices because of the stronger sextupoles in the beam line. When the sextupoles are displaced horizontally and vertically, feed-downs to normal and skew quadrupole and dipole kicks are generated:

$$
\begin{gathered}
\Delta B_{x}=B \rho k_{2}[y \Delta x+x \Delta y+\Delta x \Delta y], \\
\Delta B_{y}=B \rho k_{2}\left[(x \Delta x-y \Delta y)+\frac{1}{2}\left(\Delta x^{2}-\Delta y^{2}\right)\right], \\
\Delta k_{1 \mathrm{n}}=k_{2} \Delta x, \quad \Delta k_{1 \mathrm{~s}}=k_{2} \Delta y, \\
\Delta k_{0 \mathrm{n}}=\frac{1}{2} k_{2}\left(\Delta x^{2}-\Delta y^{2}\right), \quad \Delta k_{0 \mathrm{~s}}=k_{2} \Delta x \Delta y,
\end{gathered}
$$

where $B \rho$ is the magnetic rigidity, $k_{2}$ is the normalized sextupole strength, and $k_{1 \mathrm{n}}, k_{1 \mathrm{~s}}, k_{0 \mathrm{n}}$, and $k_{0 \mathrm{~s}}$ are the normalized normal and skew quadrupole and dipole strengths, respectively. Assuming a $\frac{\pi}{2}+n \pi$ betatron phase advance between the sextupoles and the IP, the corresponding changes in the IP vertical spot size due to the feeddown to normal and skew quadrupole kicks are evaluated in [38] by

$$
\begin{gathered}
\Delta \sigma_{y}^{*}=k_{2} l_{s} \Delta x \beta_{y, s} \sigma_{y 0}^{*}, \\
\Delta \sigma_{y}^{*}=k_{2} l_{s} \Delta y \sigma_{x, s}\left|R_{34}^{s \rightarrow *}\right|,
\end{gathered}
$$

where $\beta_{x, s}$ and $\beta_{y, s}$ are the $\beta$ functions at the sextupole locations, $\sigma_{x, s}$ is the horizontal beam size at the sextupole locations, $l_{s}$ is the sextupole length, and $R_{34}^{s \rightarrow *}$ is the matrix element from the sextupole to the IP. Lowering $\beta_{y, s}$ or $k_{2}$ makes the beam line more tolerant to the transverse misalignment of the sextupoles. When the length of the FFS is reduced, the dispersion profile is shrunk accordingly as shown in Fig. 21, leading to higher sextupole gradients. Figure 22 shows the average dispersion reduction $\eta_{x, s}$ at the sextupole locations and the corresponding increase of the average sextupole strength $k_{s}$ for shorter lattices. When $L_{\mathrm{FFS}}$ is reduced from 770 to $495.7 \mathrm{~m}$, the average dispersion is reduced by $31 \%$, and the average sextupole strength is increased by $160 \%$. Figure 23 shows the tuning

TABLE VII. Luminosity performance, synchrotron radiation impact, and QD0 parameter comparisons for different FFS lengths with $L^{*}=6 \mathrm{~m}$.

\begin{tabular}{lcccccccc}
\hline \hline FFS length $[\mathrm{m}]$ & $\frac{\sigma_{x}^{*}}{\sigma_{x}^{\text {*noSR }}}$ & $\frac{\sigma_{y}^{*}}{\sigma_{y}^{* \text { noSR }}}$ & $\mathcal{L}_{\text {total }}\left[10^{34} \mathrm{~cm}^{-2} \mathrm{~s}^{-1}\right]$ & $\mathcal{L}_{1 \%}\left[10^{34} \mathrm{~cm}^{-2} \mathrm{~s}^{-1}\right]$ & $\frac{\mathcal{L}_{1 \% \%}}{\mathcal{L}_{1 \%}^{\text {noS }}}$ & $k_{\mathrm{QD0}}\left[\mathrm{m}^{-2}\right]$ & $l_{\text {QD0 }}[\mathrm{m}]$ & $\sigma_{y, \text { Oide }}[\mathrm{nm}]$ \\
\hline 770 & 1.21 & 1.35 & 6.5 & 2.14 & 0.88 & -0.0394 & 4.7 & 0.45 \\
713.3 & 1.185 & 1.44 & 6.51 & 2.12 & 0.855 & -0.0441 & 4.4 & 0.50 \\
658.9 & 1.176 & 1.56 & 6.6 & 2.11 & 0.85 & -0.0474 & 4.16 & 0.53 \\
604.5 & 1.173 & 1.59 & 6.34 & 2.01 & 0.84 & -0.0555 & 3.75 & 0.63 \\
550 & 1.155 & 1.75 & 6.37 & 2.00 & 0.80 & -0.0663 & 3.35 & 0.76 \\
495.7 & 1.15 & 1.82 & 5.93 & 1.97 & 0.79 & -0.0788 & 3.0 & 0.93 \\
\hline \hline
\end{tabular}




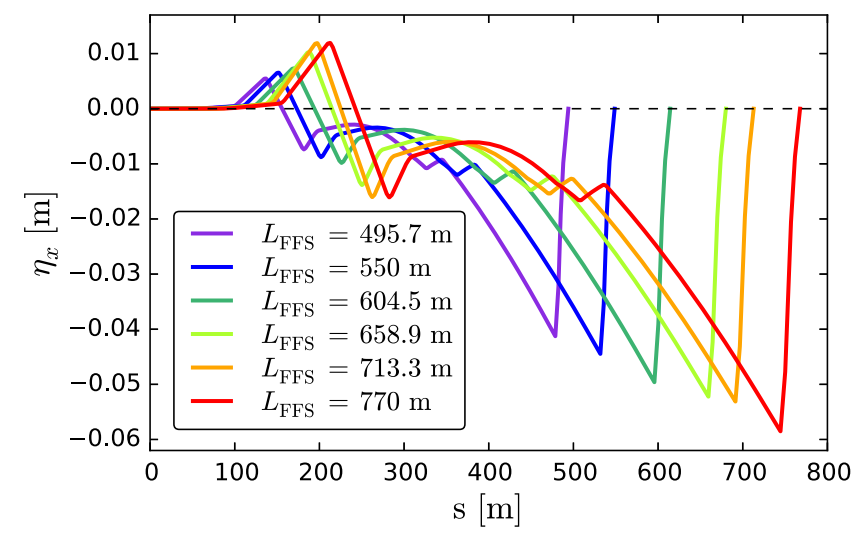

FIG. 21. Dispersion profile for different FFS lengths with $L^{*}=6 \mathrm{~m}$.

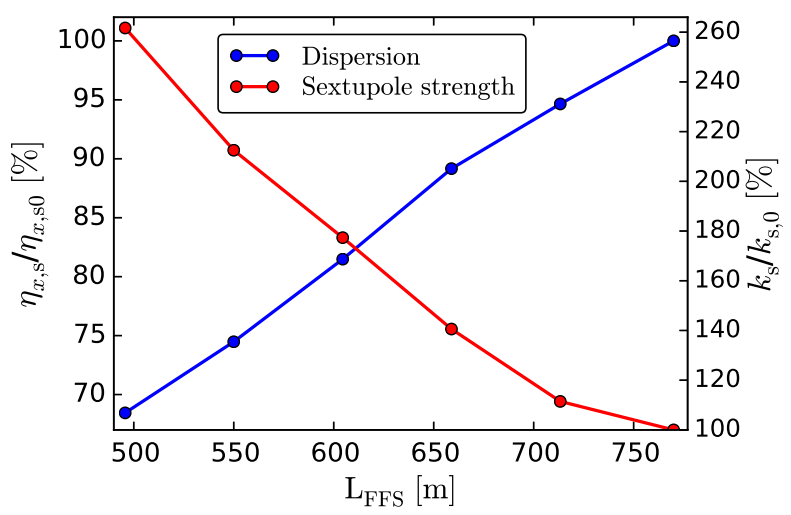

FIG. 22. Average sextupole strength and dispersion at the sextupole locations as a function of the FFS length.

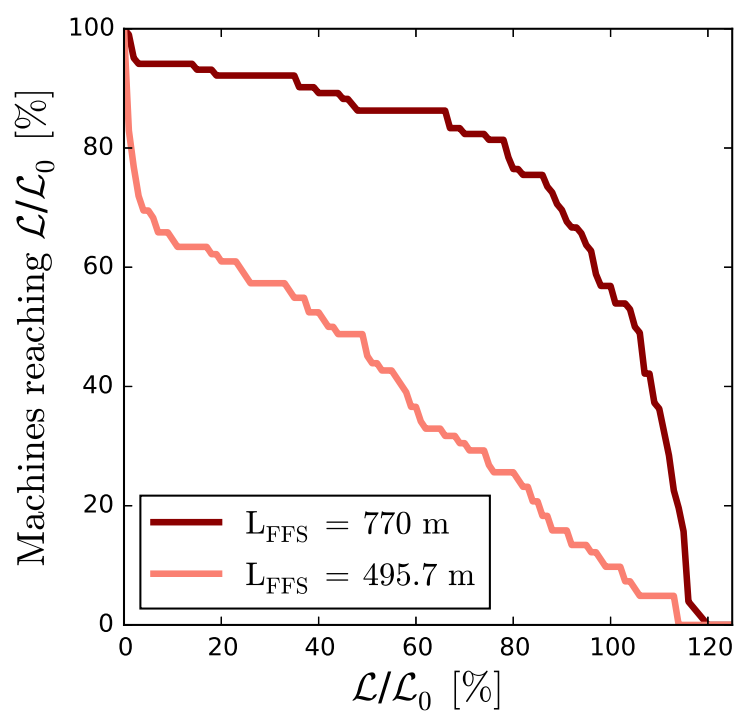

FIG. 23. Tuning efficiency comparison after one iteration of BBA and three iterations of linear knobs for $L_{\mathrm{FFS}}=495.7 \mathrm{~m}$ and $L_{\mathrm{FFS}}=770 \mathrm{~m}$ with $L^{*}=6 \mathrm{~m}$. result comparisons after one iteration of BBA and three iterations of the linear knobs, for the shortest and longest FFS lengths optimized here, with $L^{*}=6 \mathrm{~m}$. Under static transverse misalignment errors, the tuning efficiency is clearly reduced for shorter designs. For $L_{\mathrm{FFS}}=495.7 \mathrm{~m}, 16 \%$ of the machines reach $\geq 90 \%$ of $\mathcal{L}_{0}$, while $71 \%$ of the machines reach $\geq 90 \%$ of $\mathcal{L}_{0}$ for $L_{\mathrm{FFS}}=770 \mathrm{~m}$.

While the maximum luminosity achievable differs only slightly for shorter $L_{\mathrm{FFS}}$ when the beam line is optimized, one should expect a longer tuning time to reach it.

\section{Longer FFS with $L^{*}=3.5 \mathrm{~m}$}

Tuning efficiency dependence on $L_{\mathrm{FFS}}$, under transverse misalignment, has been observed also for longer systems with $L^{*}=3.5 \mathrm{~m}$ and is discussed in this section. As shown in Refs. [18,20], tuning the nominal FFS design for the local scheme requires a longer tuning time to recover the luminosity. A tuning-based design optimization has been performed on the nominal lattice. The tuning performance is thus promoted as a figure of merit, along with the luminosity of the error-free system, for the optimization of the FFS design. The strategy is to reduce the sextupole strength $k_{2}$ along the FFS in order to improve the tuning efficiency while preserving the maximum luminosity achievable. Increasing the bending magnet angles is strongly limited by the synchrotron radiation emitted at $3 \mathrm{TeV}$, and the available range of dispersion increase for which the luminosity stays within the design requirements is too small to significantly reduce $k_{2}$. For the first energy stage of CLIC at $380 \mathrm{GeV}$, this technique has been applied for the optimization of the FFS design, as the bend angles can be largely increased without a significant luminosity reduction [39]. For the final energy at $3 \mathrm{TeV}$, the dispersion has been increased with the length of the FFS. The bending magnets are lengthened and weakened accordingly in order to minimize the additional energy spread generated by the synchrotron radiation. The same optimization process described for the shorter FFS designs with $L^{*}=6 \mathrm{~m}$ has been applied for the longer designs with $L^{*}=3.5 \mathrm{~m}$ : matching of the linear optics for the lengthened FFS with scaled drift, bend, and quadrupole lengths, $L_{\text {QF1-QD0 }}$ optimization for the second-order chromaticity compensation, and dispersion optimization. This procedure has been applied on five longer systems, from 450 to $770 \mathrm{~m}$, and the optimal performances are shown in Table VIII.

For all designs, the total and peak luminosity achieved are above the design requirements with at least $19 \%$ and $15 \%$ of the luminosity budget, respectively. A similar dependence between the synchrotron radiation influence and FFS length is observed for the nominal and the long $L^{*}$ designs. The impact of the synchrotron radiation on the horizontal beam size $\sigma_{x}^{*}$ increases with the length of the 
TABLE VIII. Luminosity performance and synchrotron radiation impact comparison for different FFS lengths with $L^{*}=3.5 \mathrm{~m}$.

\begin{tabular}{|c|c|c|c|c|c|c|c|c|}
\hline FFS length [m] & $\frac{\sigma_{x}^{*}}{\sigma_{x}^{* \text { noSR }}}$ & $\frac{\sigma_{y}^{*}}{\sigma_{y}^{* \text { no SR }}}$ & $\mathcal{L}_{\text {total }}\left[10^{34} \mathrm{~cm}^{-2} \mathrm{~s}^{-1}\right]$ & $\mathcal{L}_{1 \%}\left[10^{34} \mathrm{~cm}^{-2} \mathrm{~s}^{-1}\right]$ & 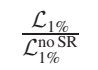 & $k_{\mathrm{QD} 0}\left[\mathrm{~m}^{-2}\right]$ & $l_{\mathrm{QD} 0}[\mathrm{~m}]$ & $\sigma_{y, \text { Oide }}[\mathrm{nm}]$ \\
\hline 770 & 1.22 & 1.21 & 7.02 & 2.34 & 0.894 & -0.053 & 4.7 & 0.50 \\
\hline 691 & 1.20 & 1.25 & 7.06 & 2.38 & 0.892 & -0.062 & 4.2 & 0.54 \\
\hline 663 & 1.188 & 1.43 & 7.2 & 2.38 & 0.86 & -0.066 & 4.0 & 0.58 \\
\hline 552 & 1.185 & 1.58 & 7.2 & 2.34 & 0.85 & -0.086 & 3.35 & 0.71 \\
\hline 497 & 1.183 & 1.59 & 7.1 & 2.33 & 0.84 & -0.10 & 3.0 & 0.80 \\
\hline 450 & 1.18 & 1.86 & 7.04 & 2.3 & 0.81 & -0.11 & 2.73 & 0.92 \\
\hline
\end{tabular}

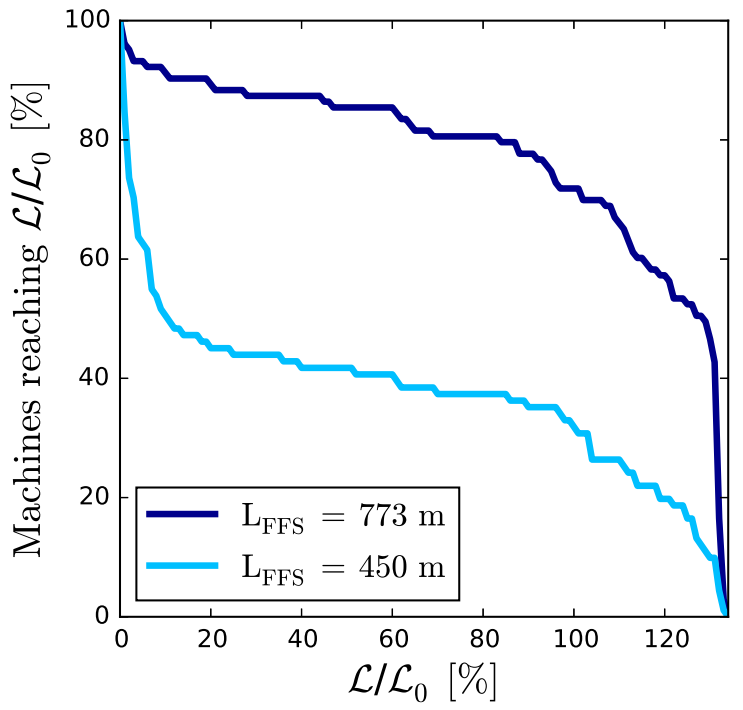

FIG. 24. Tuning efficiency after one iteration of BBA and three iterations of linear knobs for $L_{\mathrm{FFS}}=450 \mathrm{~m}$ (nominal design) and $L_{\mathrm{FFS}}=770 \mathrm{~m}$ with $L^{*}=3.5 \mathrm{~m}$.

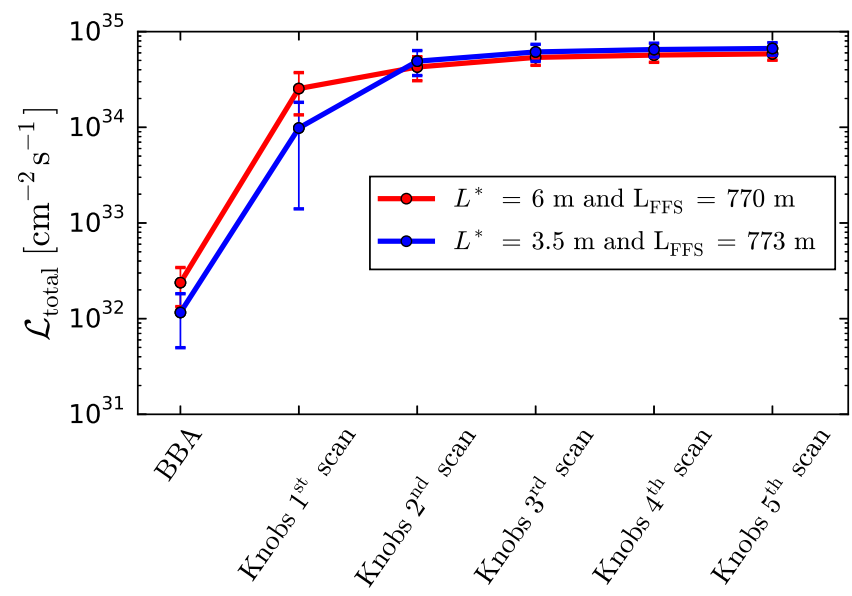

FIG. 25. Comparison of the average luminosity, over 100 machines simulated, achieved after BBA and iterations of linear knobs applied on the $L^{*}=6 \mathrm{~m}$ design and the $L^{*}=$ $3.5 \mathrm{~m}$ design with $L_{\mathrm{FFS}}=770 \mathrm{~m}$. Five scans of linear knobs were applied, which represents approximately 2000 luminosity measurements. system, while the impact on the vertical beam size and luminosity is reduced. The quadrupole gradient $k_{\mathrm{QD} 0}$ is reduced, and its length $l_{\mathrm{QD} 0}$ increased with $L_{\mathrm{FFS}}$, resulting in a reduction of the vertical beam size contribution from the Oide effect. The average strength of the sextupoles in the FFS has been reduced by $55 \%$ when the length of the system is increased from $L_{\mathrm{FFS}}=450 \mathrm{~m}$ to $L_{\mathrm{FFS}}=770 \mathrm{~m}$. In Fig. 24, the tuning efficiency is compared for the $L_{\mathrm{FFS}}=450 \mathrm{~m}$ and $L_{\mathrm{FFS}}=770 \mathrm{~m}$ design options after one iteration of BBA and three iterations of linear knobs applied to 100 machines with transversely misaligned elements. As for the long $L^{*}$ designs, the tuning performances are improved for longer FFS for $L^{*}=3.5 \mathrm{~m}$. For the nominal design with $L_{\mathrm{FFS}}=450 \mathrm{~m}, 35 \%$ of the machines reach $\geq 90 \%$ of $\mathcal{L}_{0}$, while for $L_{\mathrm{FFS}}=770 \mathrm{~m}$, $78 \%$ of the machines reach $\geq 90 \%$ of $\mathcal{L}_{0}$. Scans of linear knobs were applied to the latter FFS length option. After only five scans, the tuning efficiency approaches the goal, similarly as for the $L^{*}=6 \mathrm{~m}$ design with $L_{\mathrm{FFS}}=770 \mathrm{~m}$ for the same number of iterations, with $82 \%$ of the machines reaching at least $90 \%$ of $\mathcal{L}_{0}$. The evolution of the average luminosity recovered after each tuning step for both $L^{*}$ options is shown in Fig. 25.

\section{CONCLUSIONS}

An optimized BDS with a longer $L^{*}$ allowing a simplified version of the MDI has been addressed for the CLIC at $3 \mathrm{TeV}$. The differences in the total and in the $1 \%$ energy peak luminosities from the short $L^{*}$ design BDS compared with the alternative $L^{*}=6 \mathrm{~m}$ one are estimated to be $11.5 \%$ and $10.7 \%$, respectively. It is worth mentioning that the luminosity difference between these two $L^{*}$ options does not include the impact of the solenoid and QD0 stabilization on the integrated luminosity, which are expected to be largely reduced for the $L^{*}=6 \mathrm{~m}$ option. The tuning campaign conducted on the long $L^{*}$ FFS has demonstrated its feasibility to the same level or better than the $L^{*}=3.5 \mathrm{~m}$ lattice in the CDR with $87 \%$ of the machines that reach at least $110 \%$ of the design luminosity requiring approximately 5000 luminosity measurements. In addition, shorter designs have been fully optimized, offering slightly lower performances that nonetheless fulfill the design requirements while reducing tunnel costs. However, 
one should expect a longer tuning time, due to the stronger sextupoles, and a possible tightening of the collimation gaps, due to the smaller apertures needed for the FD. The scaled FFS, with $L^{*}=6 \mathrm{~m}$ and a length of $770 \mathrm{~m}$, remains the best option in terms of luminosity and tuning performance, which make it a realistic and robust candidate for the future CLIC BDS. The evaluation of the detector performance increase from a larger acceptance of the quadrupole-free detector option will be decisive for the choice of the CLIC FFS $L^{*}$ design.

\section{ACKNOWLEDGMENTS}

We acknowledge Konrad Elsener and the CLICdp team for providing the layout illustration of the new detector model, Benoit Curé for the computation of the detector fields, Phil Burrows for his comments on the IP feedback, and Lau Gatignon for his coordination of the MDI working group. Thanks also to Daniel Schulte, Hector Garcia Morales, and Jonas Breunlin for their useful discussions and comments.

[1] R. Tomás, Overview of the Compact Linear Collider, Phys. Rev. ST Accel. Beams 13, 014801 (2010).

[2] CLIC conceptual design report, Vol. 1, 2012.

[3] P. Raimondi and A. Seryi, Novel final focus design for future linear colliders, Phys. Rev. ST Accel. Beams 86, 3779 (2003).

[4] CLIC conceptual design report, Vol. 2, 2012.

[5] L. Gatignon, Report No. CLIC-Note-937, 2012.

[6] Y. Nosochkov and A. Seryi, Compensation of detector solenoid effects on the beam size in a linear collider, Phys. Rev. ST Accel. Beams 8, 021001 (2005).

[7] R. B. Appleby and B. Dalena, Report No. CLIC-Note-886, 2011.

[8] Y. Inntjore Levinsen and B. Dalena, Impact of detector solenoid on the Compact Linear Collider luminosity performance, Phys. Rev. ST Accel. Beams 17, 051002 (2014).

[9] A. Seryi, in Proceedings of the CLIC08 Workshop, 2008 (unpublished).

[10] CLIC Report No. Note-776, 2008.

[11] D. Angal-Kalinin, B. Dalena, J. L. Fernandez Hernando, F. Jackson, E. Marin Lacoma, R. Mutzner, J. Resta Lopez, G. Rumolo, D. Schulte, A.Seryi, R. Tomas Garcia, and G. Zamudio, Report No. CERN-ATS-2010-088, 2010.

[12] G. Zamudio and R. Tomás, Report No. CLIC-Note-882, 2010.

[13] E. Marin and R. Tomás, Report No. CERN-THESIS-2012218, 2012.

[14] CLICdp Collaboration, Report No. CLICdp-Note-2017001, 2017.

[15] L. Gatignon, in Proceedings of CLIC Workshop 2016 (unpublished).
[16] P. Tenenbaum and T. O. Raubenheimer, A dispersion-free trajectory correction technique for linear colliders, Nucl. Instrum. Methods Phys. Res., Sect. A 302, 191 (1991).

[17] A. Latina, R. Tomás, and D. Schulte, Report Nos. CERNAB-2008-011, CLIC-Note 753, 2008.

[18] B. Dalena, J. Barranco, A. Latina, E. Marin, J. Pfingstner, D. Schulte, J. Snuverink, R. Tomás, and G. Zamudio, BDS tuning and luminosity monitoring in CLIC, Phys. Rev. ST Accel. Beams 15, 051006 (2012).

[19] H. Grote and F. Schmidt, Report No. CERN-AB-2003-024, 2003.

[20] H. Garcia and R. Tomás, Final-focus systems for multiTeV linear colliders, Phys. Rev. ST Accel. Beams 17, 101001 (2014).

[21] R. Tomás, Report No. CERN-AB-Note-017, 2006.

[22] R. Tomás, Nonlinear optimization of beam lines, Phys. Rev. ST Accel. Beams 9, 081001 (2006).

[23] D. Martínez, A. Rosam, R. Tomás, and R. de Maria, Report No. CERN-ATS-Note-2012-087 TECH, 2012.

[24] E. Forest, F. Schmidt, and E. McIntosh, Report Nos. CERN-SL-2002-044, KEK-Report 2002-3, 2002.

[25] E. D'Amico, G. Guignard, N. Leros, and D. Schulte, Report No. CERN/PS 2001/028, 2001.

[26] D. Schulte, Report No. CERN-PS-99-014-LP, Report No. CLIC-Note-387, 2009.

[27] D. Schulte, in Proceedings of the 9th International School for Linear Colliders, 2015 (unpublished).

[28] R. Tomás, H. Braun, M. Jorgensen, and D. Schulte, in Proceedings of the 11th European Particle Accelerator Conference, Genoa, 2008 (EPS-AG, Genoa, Italy, 2008), OPP038.

[29] H. Mainaud Durand, D. Missiaen, and G. Stern, in Proceedings of the 4th International Particle Accelerator Conference, IPAC-2013, Shanghai, China, 2013 (JACoW, Shanghai, China, 2013), WEPME046.

[30] Y. Chung, G. Decker, and K. Evans, Jr., in Proceedings of the 15th Particle Accelerator Conference, PAC1993, Washington, DC, 1993 (IEEE, New York, 1993), pp. 2263-2265.

[31] C. Fischer and G. Parisi, Report No. CERN-SL-96-065-BI, CLIC Note 315, 1996.

[32] Y. Nosochkov, P. Raimondi, T. O. Raubenheimer, A. Seryi, and M. Woodley, in Proceedings of the 8th European Particle Accelerator Conference, Paris, 2002 (EPS-IGA and CERN, Geneva, 2002).

[33] T. Okugi, S. Araki, P. Bambade, K. Kubo, S. Kurado, M. Masuzawa, E. Marin, T. Naito, T. Tauchi, N. Terunuma, R. Tomás, J. Urakawa, G. White, and M. Woodley, Linear and second order optics corrections for the KEK Accelerator Test Facility final focus beam line, Phys. Rev. ST Accel. Beams 17, 023501 (2014).

[34] J. Snuverink, J. Barranco, H. Garcia, Y. Levinsen, R. Tomás, and D. Schulte, in Proceedings of the 4th International Particle Accelerator Conference, IPAC2013, Shanghai, China, 2013 (JACoW, Shanghai, China, 2013), TUPME051.

[35] O. Blanco, R. Tomás, and P. Bambade, in Proceedings of the 5th International Particle Accelerator Conference, 
IPAC14, Dresden, Germany, 2014 (JACoW, Geneva, 2014), TUPME005.

[36] K. Oide, Synchrotron-radiation limit on the focusing of electron beams, Phys. Rev. ST Accel. Beams 61, 15 (1988).

[37] O. Blanco, R. Tomás, and P. Bambade, Beam focusing limitation from synchrotron radiation in two dimensions, Phys. Rev. Accel. Beams 19, 021002 (2016).
[38] F. Zimmermann, Lecture Notes for Accelerator Physics and Technologies for Linear Colliders (Publisher, location, 2002), p. 575.

[39] F. Plassard, A. Latina, E. Marin, D. Schulte, R. Tomás, and P. Bambade, in Proceedings of the 8th International Particle Conference, IPAC17, Copenhagen, Denmark, 2017 (JACoW, Geneva, 2017), MOPIK100. 